International Mathematical Forum, 2, 2007, no. 23, 1111 - 1118

\title{
Harmonic Curvatures of Null Curves and the Null Helix in $R_{1}^{m+2}$
}

\author{
Ayșe Altın \\ Hacettepe University \\ Science Faculty, Mathematics Department \\ 06532 Beytepe Ankara, Turkey \\ ayse@hacettepe.edu.tr
}

\begin{abstract}
In this work we calculated the Frenet apparatus of a null curve $C$ in $R_{1}^{m+2}$ in terms of the Frenet apparatus of the curve $C^{*}$ which is the orthogonal projection of $C$ on $R^{m+1}$. The harmonic curvatures of $C$ are defined. We also give the theorems which provides some information about a null helix. If $C$ is a null helix then it must be contained in a four dimensional subspace in $R_{1}^{m+2}$.
\end{abstract}

Mathematics Subject Classification: 53A, 53C

Keywords: Semi-Riemannian manifold, null curve, harmonic curvatures, null helix. the pitch, the angle of pitch

\section{Preliminary}

The smooth curve $C=\alpha(I)$ in a semi-Riemannian manifold $\left(M^{m+2}, g\right)$ is said to be a null curve if the velocity vector to $C$ at any point is a null vector.

Let $T C$ be the tangent bundle of $C$ and $T C^{\perp}=\cup_{t \in I} T_{\alpha(t)} C^{\perp}$, where $T_{\alpha(t)} C^{\perp}=\left\{V_{\alpha(t)} \in T_{\alpha(t)} M: g\left(V_{\alpha(t)}, \alpha^{\prime}(t)\right)=0\right\}$.

At each point $\alpha(t)$, we choose a complementary vector space to $T_{\alpha(t)} C$ in $T_{\alpha(t)} C^{\perp}$. Denote by $S\left(T_{\alpha(t)} C^{\perp}\right)$, this chosen subspace. Hence, we get a vector bundle $S\left(T C^{\perp}\right)$ on $\alpha$. Since

$$
T C^{\perp}=T C \perp S\left(T C^{\perp}\right),
$$

$S\left(T C^{\perp}\right)$ is a vector bundle of rank $\mathrm{m}$. The non-degenerate vector bundle $S\left(T C^{\perp}\right)$ is called a screen vector bundle of $C$. Therefore we have 


$$
\left.T M\right|_{C}=S\left(T C^{\perp}\right) \perp S\left(T C^{\perp}\right)^{\perp}
$$

where $S\left(T C^{\perp}\right)^{\perp}$ is a complementary orthogonal vector bundle to $S\left(T C^{\perp}\right)$ in $\left.T M\right|_{C}$.

Theorem 1.1. Let $C$ be a null curve of a proper semi-Riemannian manifold $(M, g)$ and $S\left(T C^{\perp}\right)$ be a screen vector bundle of $C$. Then there exists a unique vector bundle $n \operatorname{tr}(C)$ over $C$ of rank 1 , such that on each coordinate neighbourhood $U \subset C$ there is a unique $N \in \Gamma\left(\left.n \operatorname{tr}(C)\right|_{U}\right)$ satisfying

$$
g\left(\alpha^{\prime}(t), N\right)=1, \quad g(N, N)=0, \quad g(N, X)=0, \quad \forall X \in \Gamma\left(\left.S\left(T C^{\perp}\right)\right|_{U} .\right.
$$

Consider

$$
\operatorname{tr}(C)=n \operatorname{tr}(C) \perp S\left(T C^{\perp}\right),
$$

from (1), (2) then we have the following sum

$$
\left.T M\right|_{C}=T C \oplus \operatorname{tr}(C)=(T C \oplus n \operatorname{tr}(C)) \perp S\left(T C^{\perp}\right) .
$$

The vector field $N$, which was constructed in this theorem, is said to be the null transversal vector field of $C$ with respect to $\alpha^{\prime}$ [2.p.53]. form

A null curve $C$ in $R_{1}^{m+2}$ is given locally by the equation of the following

$$
\alpha(s)=\left(s, \quad \int_{0}^{s} \alpha_{1}, \ldots, \int_{0}^{s} \alpha_{m+1}\right),
$$

where, $\quad \alpha_{1}=\operatorname{cosb}_{1}(s) d s+c_{1}, \quad \alpha_{a}=\operatorname{cosb}_{a}(s) \prod_{k=1}^{a-1} \operatorname{sinb}_{k}(s) d s+c_{a}$, $a \in\{2, \ldots, m\}, \quad \alpha_{m+1}=\prod_{k=1}^{m} \operatorname{sinb}_{k}(s) d s+c_{m+1}, \quad \mathrm{c}_{k} \in R$, $b_{k}$ are smooth functions for any $k \in\{1, \ldots, m\}$, and $s$ is the arc-length of the orthogonal projection $C^{*}=\alpha^{*}(I)$ of $C$ on $R^{m+1}$ give by, [2,p.73],

$$
\alpha^{*}(t)=\left\{\alpha_{1}^{*}(t), \alpha_{2}^{*}(t), \ldots, \alpha_{m+1}^{*}(t)\right\} .
$$

By the ideas in $[4, p .73]$ and $[5, p .160]$, a helix is defined as a curve which has a constant scalar product of its tangent vector field and a constant vector field.

In this paper, we mean by $\alpha^{l}$ s,,$\quad(1 \leq l \leq r)$ the derivatives of the curve $\alpha$.

Let $\alpha^{*}$ be a regular curve in $R^{m+1}$ and $\psi=\left\{\left(\alpha^{*}\right)^{\prime}(t),\left(\alpha^{*}\right)^{\prime \prime}(t), \ldots, \alpha^{* r}(t)\right\}$ be a maximal linearly independent set. The orthonormal system $\left\{V_{1}(t), V_{2}(t), \ldots, V_{r}(t)\right\}$ can be obtained from $\psi$. This is called a Frenet frame at the point $\alpha^{*}(t),[3]$. 
Defnition 1.2. Let $\alpha^{*}$ be a regular curve in $R^{m+1}$ and $\left\{V_{1}(t), V_{2}(t), \ldots, V_{r}(t)\right\}$ be the Frenet frame at the point $\alpha^{*}(t)$.

The functions $k_{i}: I \longrightarrow R$ defined by

$$
k_{i}(t)=g\left(V_{i}^{\prime}(t), V_{i+1}(t)\right), \quad 1 \leq i \leq r-1
$$

are called curvature functions on $\alpha^{*}$. Moreover, the real number $k_{i}(t)$ is called the $i-t h$ curvature on $\alpha^{*}$ at the point $\alpha^{*}(t)$.

Let $\alpha^{*}$ be a unit speed curve in $R^{m+1}$ and the set $\left\{V_{1}(t), V_{2}(t), \ldots, V_{r}(t)\right\}$ be the Frenet frame at the point $\alpha^{*}(t)$. Then, the followings hold, [6,p.194],

$$
\begin{aligned}
V_{1}^{\prime}(t) & =k_{1}(t) V_{2}(t) \\
V_{i}^{\prime}(t) & =-k_{i-1}(t) V_{i-1}(t)+k_{i}(t) V_{i+1}(t), \quad i<i<r \\
V_{r}^{\prime}(t) & =-k_{r-1}(t) V_{r-1}(t) .
\end{aligned}
$$

\section{HELIX AND HARMONIC CURVATURES}

Theorem 2.1. Let $\alpha$ and $\alpha^{*}$ be the curves as in equation (4) and $\left\{V_{1}, V_{2}, \ldots, V_{r}\right\}$, $\left\{k_{1}, k_{2}, \ldots, k_{r-1}\right\}$ be Frenet fields and curvature functions of curve $\alpha^{*}$ respectively. Then, $\alpha$ is a null curve in $R_{1}^{r+1}$. More over if we choose $S\left(T C^{\perp}\right)$ spanned by $\left\{W_{2}, \ldots, W_{r}\right\}$, then we have the null transversal vector field $N=\frac{1}{2}\left(-1, V_{1}\right)$, and the Frenet equations are

$$
\begin{aligned}
& \alpha^{\prime \prime}=k_{1} W_{2}, \\
& N^{\prime}=\frac{1}{2} k_{1} W_{2}, \\
& \left(W_{2}\right)^{\prime}=-\frac{1}{2} k_{1} W_{0}-k_{1} N+k_{2} W_{3}, \\
& \left(W_{3}\right) \prime=-k_{2} W_{2}+k_{3} W_{4}, \\
& \left(W_{4}\right)^{\prime}=-k_{3} W_{3}+k_{4} W_{5}, \\
& \ldots \ldots \ldots \ldots \ldots \ldots \ldots \ldots \ldots \ldots \ldots \ldots \ldots \ldots \ldots \ldots \ldots \\
& \left(W_{r-1}\right)^{\prime}=-k_{r-2} W_{r-2}+k_{r-1} W_{r}, \\
& \left(W_{r}\right)^{\prime}=-k_{r-1} W_{r-1}
\end{aligned}
$$

where

$$
W_{j}=\left(0, V_{j}\right) \quad j \in\{2, \ldots, r\}
$$

and the Frenet frame is $F=\left\{\alpha^{\prime}, N, W_{2}, \ldots, W_{r}\right\}$ on $R_{1}^{r+1}$ along $\alpha$.

Proof. From (4) we have

$$
\alpha^{\prime}=\left(1,\left(\alpha^{*}\right)^{\prime}\right), \quad \alpha^{j}=\left(0,(\alpha *)^{j}\right), \quad j \geq 2 .
$$


Therefore $\left\{\alpha^{\prime}, \alpha^{\prime \prime}, \ldots, \alpha^{r}\right\}, \quad r \leq m+1$ is the maximal linearly independent set. Since $\alpha^{*}$ has at most (r-1) non zero curvatures, $\alpha^{*}$ is contained in $R^{r}$. Therefore $\alpha$ is also contained in $R_{1}^{r}$.

We choose $S\left(T C^{\perp}\right)=\operatorname{span}\left\{W_{2}, \ldots, W_{r}\right\}$ and a complementary vector bundle $H$ to $T C$ in $S\left(T C^{\perp}\right)$,

$Y=\left(0,\left(\alpha^{*}\right)^{\prime}\right) \in \Gamma\left(\left.H\right|_{U}\right)$ and $g\left(\alpha^{\prime}, Y\right) \neq 0$ on $U$. We calculated the null transversal vector field and found that

$$
N=\frac{1}{g\left(\alpha^{\prime}, Y\right)}\left\{Y-\frac{g(Y, Y)}{2 g\left(\alpha^{\prime}, Y\right)} \alpha^{\prime}\right\}=\frac{1}{2}\left(-1,\left(\alpha^{*}\right)^{\prime}\right) .
$$

Since $\left(\alpha^{*}\right)^{\prime}=V_{1}$, we have

$$
\alpha^{\prime}=\left(1, V_{1}\right), \quad N=\frac{1}{2}\left(-1, V_{1}\right) .
$$

We differentiate (10), (11) and by using (6), (7), (8) we obtain (9).

We now give the definition of a null helix in semi-Euclidean space $R_{1}^{m+2}$ in a similar way to [1], as follows.

Definition 2.2. Let $\alpha$ be a null curve in $R_{1}^{m+2}$ and $X$ be a non zero constant vector field. If

$$
g\left(\alpha^{\prime}(t), X\right)=\text { constant } \neq 0, \quad \text { for all } t \in I,
$$

then, $\alpha$ is said to be a null helix in $R_{1}^{m+2}$ and $\operatorname{span}\{X\}$ is said to be the inclination axes of $\alpha$,

Example 2.3. Let $a, \sigma, \rho$ be non-zero constants, $b$ be constant and let $\alpha: R \longrightarrow R_{1}^{3}$ be the curve difened by

$$
\alpha(t)=\left(a t+b, \frac{1}{\rho} \sigma \cos \rho t, \frac{1}{\rho} \sigma \sin \rho t\right), \quad X=(1,0,0)
$$

where $a^{2}=\sigma^{2}$.

Example 2.4. $\alpha: R \longrightarrow R_{1}^{3}$ be the curve difened by

$$
\alpha(t)=\left(\frac{4}{3} t^{3}+t, \quad 2 t^{2}, \quad \frac{4}{3} t^{3}-t\right), \quad X=(1,0,1)
$$

Example 2.5. Let $a, \sigma, \rho$ be non-zero constants, $b, c, d$ be constants and let $\alpha: R \longrightarrow R_{1}^{4}$ be the curve difened by

$$
\alpha(t)=\left(a t+b, \quad c t+d, \quad \frac{1}{\rho} \sigma \cos \rho t, \quad \frac{1}{\rho} \sigma \sin \rho t\right), \quad X=(1,0,0,0)
$$


where $a^{2}=c^{2}+\sigma^{2}$.

Example 2.6. Let $a, \sigma, \rho, \omega, d$ be non-zero constants, $b$ be constant and let $\alpha: R \longrightarrow R_{1}^{5}$ be the curve difened by

$\alpha(t)=\left(a t+b, \frac{1}{\rho} \sigma \cos \rho t, \frac{1}{\rho} \sigma \sin \rho t, \frac{1}{\omega} d \cos \omega t, \frac{1}{\omega} d \cos \omega t\right), \quad X=(1,0,0,0,0)$

where $a^{2}=\sigma^{2}+d^{2}$.

Definition 2.7. Suppose that $k_{1}, k_{2}, \ldots, k_{n-1}$ are curvature functions of a curve $\alpha$. A function $H_{i}: I \longrightarrow R$ defined by

$$
H_{i}(t)=\left\{\begin{array}{lrr}
\frac{k_{1}(t)}{k_{2}(t)}, & \text { if } & i=1 \\
\frac{1}{k_{i+1}(t)}\left\{H_{i-1}^{\prime}(t)+k_{i}(t) H_{i-2}(t)\right\}, & \text { if } & 2 \leq i \leq n-2
\end{array}\right.
$$

is called the $i-t h$ harmonic curvature function of $\alpha$.

Theorem 2.8. Let $\alpha$ be a null helix in $R_{1}^{m+2}$, and $\operatorname{span}\{X\}$ be the inclination axes of $\alpha$. If $\left\{\alpha^{\prime}, N, W_{2}, \ldots, W_{r}\right\}$ is the Frenet frame fields of $\alpha$ and $H_{1}, H_{2}, \ldots, H_{r-2}$ are harmonic curvature functions of $\alpha$, then

$$
g\left(W_{i+2}, X\right)=H_{i} g\left(\frac{\alpha^{\prime}+2 N}{2}, X\right), \quad 1 \leq i \leq r-2 .
$$

Proof. We use induction on i. Since $\alpha$ is null helix, then $g\left(\alpha^{\prime}, X\right)=$ constant. If we take derivative, then we have $<\alpha^{\prime \prime}, X>=0$. Thus by using (6), we obtain

$$
g\left(W_{2}, X\right)=0
$$

Taking derivative in (14) and using (7), we get

$$
g\left(W_{3}, X\right)=H_{1}\left(\frac{\alpha^{\prime}+2 N}{2}, X\right) .
$$

Hence (13) is true for $i=1$. By the induction hypothesis, we have

$$
g\left(W_{i+1}, X\right)=H_{i-1} g\left(\frac{\alpha^{\prime}+2 N}{2}, X\right) .
$$

Using (15) and (7) we get

$$
g\left(W_{i+2}, X\right)=\frac{1}{k_{i+1}}\left\{k_{i} g\left(W_{i}, X\right)+\left(H_{i-1}\right)^{\prime} g\left(\frac{\alpha^{\prime}+2 N}{2}, X\right)\right\} .
$$


By the induction hypothesis and by (16), we have

$$
g\left(W_{i+2}, X\right)=\frac{1}{k_{i+1}}\left\{k_{i} H_{i-2}+\left(H_{i-1}\right)^{\prime}\right\} g\left(\frac{\alpha^{\prime}+2 N}{2}, X\right) .
$$

Therefore, (13) follows from (12).

Lemma 2.9. Let $\alpha$ be a null helix in $R_{1}^{m+2}, \operatorname{span}\{X\}$ be the inclination axes, $\left\{\alpha^{\prime}, N, W_{2}, \ldots, W_{r}\right\}$ be the Frenet frame fields of $\alpha$ and let $\Pi$ be the orthogonal projection of $R_{1}^{m+2}$ onto the space $\operatorname{span}\left\{\alpha^{\prime}, N, W_{2}, \ldots, W_{r}\right\}$. If $r<$ $m$, then $\operatorname{span}\{\Pi(X)\}$ is the inclination axes in $\operatorname{span}\left\{\alpha^{\prime}, N, W_{2}, \ldots, W_{r}\right\}$.

Proof. We can choose $\left\{\alpha^{\prime}, N, W_{2}, \ldots, W_{r}, w_{1}, \ldots, w_{m-r}\right\}$ as an quasi-orthonormal basis of $R_{1}^{m+2}$. In this case

$$
X=x_{0} \alpha^{\prime}+x_{1} N+\sum_{i=2}^{r} x_{i} W_{i}+\sum_{j=1}^{m-r} b_{j} w_{j}, \quad \Pi(X)=x_{0} \alpha^{\prime}+x_{1} N+\sum_{i=2}^{r} x_{i} W_{i} .
$$

Since $g\left(\sum_{j=1}^{n-r} b_{j} w_{j}, \alpha^{\prime}\right)=0$ and $g\left(X, \alpha^{\prime}\right)=$ constant $\neq 0$, we have

$$
g\left(x_{0} \alpha^{\prime}+x_{1} N+\sum_{i=2}^{r} x_{i} W_{i}, \alpha^{\prime}\right)=\text { constant } \neq 0
$$

Since $\operatorname{span}\{X\}$ is inclination axes, then $\Pi(X)$ is also non zero and constant.

Theorem 2.10. Let $\alpha$ be a curve in $R_{1}^{m+2}$ with the Frenet frame field $\left\{\alpha^{\prime}, N, W_{2}, \ldots, W_{r}\right\}$ and with harmonic curvatures $H_{1}, H_{2}, \ldots, H_{r-2}, r \leq m$. Then, $\alpha$ is a null helix in $R_{1}^{m+2}$ if and only if $H_{i}$ 's are constant and $x_{1} \neq 0$.

Proof. Let $\operatorname{span}\{X\}$ be the inclination axes of $\alpha$. Thus

$$
g\left(\alpha^{\prime}, X\right)=\text { constant } \neq 0 \quad \text { and } \quad g\left(\alpha^{\prime}, \Pi(X)\right)=\text { constant } \neq 0 .
$$

Using (14) and (15), we obtain

$$
\Pi(X)=x_{0} \alpha^{\prime}+x_{1} N+\sum_{i=3}^{r} H_{i-2} g\left(\frac{\alpha^{\prime}+2 N}{2}, X\right) W_{i}
$$

Since $\Pi(X)$ is a non zero constant vector field, $H_{i}$ 's must be constant and $x_{1} \neq 0$.

Conversely, choose a constant vector field, $X=\alpha^{\prime}+N+\sum_{i=3}^{r} H_{i-2} W_{i}$. Then $g\left(\alpha^{\prime}, X\right)=2$. Therefore $\alpha$ is a helix.

Theorem 2.11. There is a relation between curvatures and harmonic curvatures of a curve in $R_{1}^{m+2}$ as follows. 


$$
k_{r}=\frac{\left(\sum_{i=1}^{r-2} H_{i}^{2}\right)^{\prime}}{2 H_{r-1} H_{r-2}}, \quad 3 \leq r \leq m-1
$$

Proof. We use induction on r. By (12),

$$
k_{i+1}=\frac{\left(H_{i-1}\right)^{\prime}+k_{i} H_{i-2}}{H_{i}}, \quad 2 \leq i \leq m-2 .
$$

For $i=2, k_{3}=\frac{\left(H_{1}^{2}\right)^{\prime}}{2 H_{1} H_{2}}$ and the statement (17) is true for $r=3$.

The induction hypothesis states that

$$
k_{p+1}=\frac{\left(\sum_{i=1}^{p-1} H_{i}^{2}\right)^{\prime}}{2 H_{p} H_{p-1}}, \quad p<m-1 .
$$

Using (18) and (19), we get

$$
k_{p+2}=\frac{\left(\sum_{i=1}^{p} H_{i}^{2}\right)^{\prime}}{2 H_{p+1} H_{p}}
$$

which completes the proof.

Consequently, combining (12), (17) and theorem 2.10 we can give our main theorem.

Theorem 2.12. The curve $\alpha$ is a null helix in $R_{1}^{m+2}$ if and only if $\mathrm{n} \geq 4$ and $k_{j}=0$ for $j \geq 3$.

As a consequence of this theorem we obtain the following.

Corollary 2.13. if $\alpha$ is null helix then $\alpha$ is contained in a four dimensional subspaces in $R_{1}^{m+2}$.

\section{References}

[1] A. ALTIN, Harmonic Curvatures of Non Null Curves and the Helix in $R_{v}^{n}$, Hacettepe Bulletin of Natural Sciences and Engineering. Vol 30 (2001) p.55-61.

[2] K. L. DUGGAL and A. BEJANCU, Lightlike Submanifolds of SemiRiemannian Manifolds and Applications, Kluwer Academic Publishers Group, Dordrecht, The Netherlands, 1996. 
[3] H. GLUK, Higher Curvatures of curves in Euclidean space, American Mth. Month. Vol 73 (1966), p.699-704.

[4] A. GOETZ, Introduction to Differential Geometry, Addison Wesley Publishing Company, 1970.

[5] H. W. GUGGENHEIMER, Differential Geometry, Mc Graw-Hill Book Company, inc, 1963.

[6] H.H. HACISALİHOĞLU, Diferensiyel Geometri, İnönü Üniversitesi Fen Edebiyat Fakültesi Yayinlari, 1983.

Received: May 29, 2006 